www.conferenceie.ase.ro

\title{
HIERARCHICAL TEMPORAL MEMORIES IN SECURITY INFORMATION AND EVENTS MANAGEMENT SYSTEMS
}

\author{
Mihail CAZACU \\ Bucharest University of Economic Studies, Romania \\ mihail.cazacu@gmail.com \\ Constanţa-Nicoleta BODEA \\ Bucharest University of Economic Studies, Romania \\ bodea@ase.ro
}

\begin{abstract}
Process mining techniques assist cybersecurity specialists in better understand the processes which are running in a network. Automatic process filtering represents an important component of process mining, but the lack of common sense/grounding knowledge create challenges which are similar with those reported in Natural Language Processing (NLP). The paper presents how the Hierarchical Temporal Memories (HTMs) can be applied in the Security Information and Events Management Systems for the process filtering purpose. Initial experiments were conducted and some important conclusions were already achieved. The future research will aim to design hybrid solutions for process filtering, including fuzzy controllers, rough sets and self-organizing maps, in addition to HTMs.
\end{abstract}

Keywords: Process mining, process filtering, NLP, Bidirectional Encoder Representations for Transformers, Hierarchical Temporal Memories.

JEL classification: M55, O15, O33

DOI: $10.24818 / \mathrm{ie} 2020.04 .09$

\section{Introduction}

A Security Information and Events Management System (SIEM) typically consists of two subsystems: Security Information Management System, which collects, indexes and stores logs and Security Events Management System, which monitors continuously the information collected, looking for patterns which might indicate a cybersecurity incident and signaling the incidents to the cybersecurity analysts ("rising alerts"). The patterns indicating a possible security incident are usually identified outside SIEMs, by specialized security software (antivirus, Intrusion Detection/Prevention Systems, firewalls, etc). The typical activities of cybersecurity specialists are: monitoring the SIEM reports; react to alerts by investigating them (by use SIEM's log-exploring toolset to understand which specific log entries had generated the alert and to find other correlations to the events which have generated the alert in order to decide it it's a security incident or a false positive), act on the conclusion from investigation. The main responsibility is to discriminate between cybersecurity incidents and false positives. Operations performed by cybersecurity specialists are usually logged for compliance reasons (to prevent "inside jobs" and to limit the legal liability in case of damage done by an incident to the client's system). This creates an opportunity to use Process Mining techniques (Process Discovery) to automatically identify the high-level processes (human activities) performed when investigating an alert. The challenge is to separate (filter) trial-and-error actions from the end-to-end method of dealing with that incident.

Process discovery and process conformance checking are the main types of process mining activities which are relevant for SIEMs. While process mining tools transform the vast quantity 
of log records into human-intelligible high-level business processes, the main log-processing tool used in SIEMs is not a process-mining tool.

\section{Process Filtering challenges}

In logs, process traces are represented as a succession of time-stamped records. The steps which led to dead-ends are interspersed among the steps which led to the solution. Back-tracking is not obvious and therefore automatic mechanisms for detecting it are necessary.

Not entire processing is capture on logs, due to the fact that many processes are performed by specialists, as cognitive processes. Traces in the logs may show the analyst dropped the use of a tool or the investigation of a log and started to use another tool or to investigate another log. Without any apparent recorded reason, the process discovery algorithm needs to be able to guess which of the two cases applies because the graph of the whole set of operations looks different from one case to another (the graph of the first process would be a simple chain, the second would have branches in it). Humans are often able to deduct when one changed one's mind by watching one's actions, thanks to the "common sense" (a mental model about how the World functions and how the beings in it act depending on the circumstances). Yet there are well documented instances when the "common sense" of some people is so lacking of relevant experiences that what other people are doing looks like magic (magicians' performances are such an example).

Automatic process filtering is facing with the lack of common sense/grounding knowledge like many other Artificial Intelligence tasks, especially those related to Natural Language Processing (NLP).

\section{Methods for addressing the lack of grounding/common sense knowledge}

Grounding/Common Sense knowledge means that each data (word, sentence, phrase, log line, element of the log line, etc) has contextual information (other data) attached to it. For humans, the context for the words, sentences and phrases of the language is the mental model of the world (most of the time) or an abstract mental model (when speaking about abstract concepts). This correspondence between a model of the World and the words which describe it allow humans to learn to speak correctly without being exposed to many examples of how to form correct sentences. Common sense even helps understand incomplete or incorrectly formulated sentences.

Computers can learn context in the absence of a model of the world. They do so by learning the distribution function of the conditional probabilities of strings of words. Many methods for learning such distribution functions are using Artificial Neural Networks (ANN) trained on large corpora of text. Two theoretical results justify the choice of using ANNs:

- A three layers feed-forward network can approximate any continuous function to any desired degree of precision [1];

- Certain Recurrent Neural Networks are Turing-complete therefore those RNNs can perform any algorithm $[2,3]$

Remarkable results are obtained using architectures based on BERT (Bidirectional Encoder Representations for Transformers) [4]. These are ANNs made of stacked modules, each module combining recurrent and convolutional layers. ANNs based on BERT are trained in unsupervised mode. That approach which solves a previously existing bottleneck - the absence of enough annotated texts (needed for supervised learning). It is also biologically plausible approach in that most of learning for live beings seems to be unsupervised. The fact BERT has managed to surpass other implementations, many times by a wide margin, using unsupervised learning might be its most important achievement. Nevertheless, just like any other statistical learning model, BERT needs a large amount of text from which to learn (8.96 TB). The original 
www.conferenceie.ase.ro

BERT model trains 340 million parameters on 16 Tensor Processing Units for 4 days $(\$ 7,000$ total cost estimated based on Google Cloud published prices in 2019). BERT's successor XLNet (also developed by a team from Google) cost $\$ 31,000$ to train (in 2019 prices). Relatively few research teams can afford to use this approach [5].

The costs, computational intensity and need for very large datasets suggest the following research questions:

- Can a less data-hungry and less computational-intensive approach be used for building context from data?

- Could such approach benefit from strong theorical guarantees (e.g. universal approximator of continuous functions)?

- Could such approach be based mainly on unsupervised learning?

- Could such approach be helpful especially in investigating the false positives (since that is the core responsibility of the people we want to help)?

- Could such approach be explainable and relatively simple to understand (since the entrylevel specialists are not expected to have very sophisticated mathematical skills)?

These research questions will be considered during the future research.

\section{Alternative approaches for BERT-based ANNs}

Based on literature review, the following approaches are considered as being promising:

- Fuzzy controllers, because they are universal approximators [6]. Also, they are computationally low-intensity even when they have to deal with many inputs. For certain types of fuzzy controllers, there are algorithms for unsupervised learning from data. Recently techniques were developed for constructing metrics over fuzzy sets $[7,8]$ allowing us to calculate distances between vectors with fuzzy elements.

- Self-Organizing Maps, because they have low-intensity requirements for computation, they are using unsupervised learning algorithms and they have the ability to interpolate many interesting continuous functions [9].

- Rough Sets, mainly because they are a powerful way to represent and deal with ambiguity in data with discrete values (like data in logs and text files). False positives are data which are indistinguishable from true positives as far as the classifier is concerned, which in rough sets parlance means they belong to the border of the rough set of positives. The most powerful rough set techniques are used in many unsupervised learning pipelines, they have low-intensity requirements for computation and there are powerful techniques combining roughs sets and fuzzy sets.

- Hierarchical Temporal Memories (HTMs) [10], because they are similar in many respects to Self-Organizing Maps but in addition to encoding the similarity between input vectors they can incorporate explicitly the concepts of succession in time, position in space or place inside a sequence. They are trained in an unsupervised way, they are low-intensity in terms of requirements for computation and the same architecture can be used for performing different sequence recognition tasks (e.g. switching form temporal to spatial sequence classifications) just by changing the method used for encoding the input vectors

Referring to HTMs, they are ANNs with a hierarchy of layers stacked on top of each other and named regions. Each region is made of sets of columns of artificial neurons. Each column is placed side by side with other columns in that region. Each artificial neuron in a column receive two categories of inputs: proximal inputs, feed-forwarded from cells in the same column and distal inputs, from cells in other Columns or even in other Regions. Proximal inputs are dependent on the current input data. Distal inputs are dependent on the previous input data (on 
the memorized sequence of input data). All inputs, outputs and weights are binary (this makes HTMs very low-intensive computationally;

The learning algorithm ensures that each time the same input vector is shown to a region, the same set of columns is activated. It also ensures that depending on what vector was shown before, the same set of cells is activated inside each activated column. Therefore the objects are memorized horizontally ("planar memory", similar to SOMs), while the sequence/ context of objects is memorized both vertically and horizontally, in a so-called "voluminal memory". When a cell inside a column is activated, it generates a distal input for other cells in other columns. Those cells are pre-activated (put in a "predictive" state). What follows is this:

- If the next input vector activates columns with pre-activated cells, only the pre-activated cells in the active columns will become active ("prediction is confirmed, this is a known sequence, next symbol is...");

- If the next vector does not activate columns with pre-activated cells, then all the cells of those newly activated columns become active. That in turns means a lot of cells in other columns become pre-activated ("this is a new sequence, the next symbol can be any symbol, get ready for it");

HTMs learning algorithm generates sparse representations: at any time only a small fraction of the cells of a Column and of the Columns of a Region are active. That 3D voluminal activation is converted into a sparse vector through serialization. HTMs need sparse vectors as inputs. The sparse vectors which are the inputs of the Region at the bottom of the hierarchy need to have a special propriety: similar objects need to be encoded into similar sparse vectors. The similarity of two sparse vectors is given by the number of bits they have in common. The learning mechanism ensures that two similar vectors would generate two similar activations. In spite of their good performance in predicting/recognizing vectors and sequences of vectors, and their low computational cost, the HTMs aren't as popular as the convolutional and recurrent networks because their success depends on finding the right method for encoding the input vectors in such a way that similar objects get encoded by sparse vectors which differ by only a few bits form each other. For instance, there is no known way to encode speech so that HTMs can process effectively voice messages (an otherwise easy task for RNNs).

\section{Experiments related to the HTMs usage in process filtering}

Main difficulty in exploiting the advantages offered by HTMs is finding encoding algorithms which generate sparse vectors that HTMs could use. Since there are more text datasets for NLP training than log datasets, we have decided to start the research by running experiments on classical NLP task of word prediction in order to investigate several intuitions, such as:

- Performance might depend on the encoding method (just like say, the ability to see depends on the quality of the eyesight);

- Performance might depend on the size of the training dataset (even though we want our systems to be as "frugal" as possible, building the "World Model" wound need data)

The assumption is that since the vocabulary of logs is much smaller than the vocabulary of natural languages, if the algorithms work well on NLP tasks they would work well also on Process Discovery tasks.

The experiment environment consisted of Anaconda environment for Python, with Jupyter notebooks, NLTK, Pandas and NumPy packages installed as default, plus additional specialized Python packages (e.g. MiniSOM for Self-Organizing Maps) for specialized processing.

The purpose of any encoder is to encode the words as vectors in such a way that it also captures the contexts the word might be used in. The purpose of a HTM Sparse Encoder (HTM SE) is to do so while complying with two requirements: sparsity (only a small fraction, like 5\% $20 \%$ of the bits of the vector are 1) and small distance between similar concepts. The concept 
of distance implies there is a metric on the vector space of words. The most popular metric used when working with HTMs is counting the bits which overlap. The more overlapped bits, the similar the two words. For the purpose of encoding common sense, the similarity we're interested in is the semantic similarity (as opposed to say, word-size similarity). In the absence of an internal physical model of the World, where similarity would be decided by comparing the objects or actions denominated by the words, the next best assumption is that similar words would be used in similar contexts. The immediate context of a word is the sentence containing it. The immediate context of a sentence is the phrase containing it. Even though there are larger conceptual structures for organizing texts (paragraphs, chapters, etc) the next context container we can rely on is the document. Not all documents are structured in chapters or paragraphs and even if they are, there's often difficult to detect automatically such structures.

In order to associate words with their context we decided to use the approach described in [11]. Based on this approach, the documents are represented $\mathrm{N}$-dimensional vectors, with $\mathrm{N}$ being the number of "distinct words" we choose to take into account. The concept of "Distinct Words" in itself can imply two classical approaches to pre-processing: word stemming and word lemmatization. The two pre-processing procedures result in different word sets for the same document, which might have consequences later on the recall and precision of the system.

Irrespective of how the document's set of words is chosen, the next step is the removal of "stop words", which are the most common words in a text (in any language which has them, the conjunctions and prepositions are always members the "stop words" set).

Even though this step is well intended for reducing the computational intensity of further processing by reducing the dimension of the vector which represents a document, "stop words" can often be essential for conveying meaning (and therefore for context representation.

The final pre-processing step is to decide how many words should be considered the dimensions of the vector representing the document and what values should be stored in the vector. Each decision might have consequences on how well the context is captured.

Some experiments were ran with top 40 most frequent words in the collection of 4,818 preprocessed documents (lemmatized and with stop-words eliminated).. All documents are scientific texts (pdf articles and books) about various topics of AI and Machine Learning, from my personal digital library. The reason for choosing such a corpus is that each of these documents were manually classified by the authors into 145 folders according to their main topic (e.g. Neural networks, Support Vector Machines, Fuzzy Logic, Rough Sets, etc), so it was relatively easy to check if the classification is correct by human standards, without having to read the document. The reason to choose the top 40 most frequent words in the collection was that a such number have led to good results in other works about document clustering. Regarding the values which go into the vectors, the rule is: for each document, $\mathrm{V}[\mathrm{k}]=$ frequency (in the document) of the k-th most frequent word (in the collection). That is a classical Bag-of-Words type of encoding documents as vectors of words.

\section{Conclusions}

Based on the experiments which were already conducted, we consider that the predictions from HTMs depend on the encoding method and on the size and diversity of the data from which the encoding scheme was learned, but so far we were not able to identify a clear indication which method of encoding is consistently better. As future work, other representations than Bag-of-Words for texts and logs (e.g. n-grams) will be used in our experiments in order to check if same methods (SOMs, Rough Sets Clustering and Fuzzy Sets Clustering) could lead to better results. Additional experiments using the output of pre-trained neural encoders (Word2Vec/Glove) are also considered Such vectors have the property the cosine distance between representations of semantically related words is small, so there might be a way to 
www.conferenceie.ase.ro

sparse-encode them for the HTM usage. Obtaining such vectors through training is computationally expensive so using them in a HTM pipeline sort-of beats the purpose of finding a low-cost good alternative to the current Deep Learning methods. An additional challenge is that were are not yet available pre-trained Word2Vec/Glove representations for logs.

\section{References}

[1] G. Cybenko (1989), Approximation by superpositions of a sigmoidal function, Math. Control Signal Systems 2, 303-314 DOI: https://doi.org/10.1007/BF02551274.

[2] H. T. Siegelman, E.D. Sontag (1992), On the computational power of neural nets, COLT '92: Proceedings of the fifth annual workshop on Computational learning theory, DOI: https://doi.org/10.1145/130385.130432.

[3] A. Graves, G. Wayne, I. Danihelka (2014), Neural Turing Machines, arXiv:1410.5401v2

[4] J. Devlin, M. W. Chang, K. Lee, K. Toutanova (2018), BERT: Pre-training of Deep Bidirectional Transformers for Language Understanding, arXiv:1810.04805v2.

[5] Syncedreview, Internet https://syncedreview.com/2019/06/27/the-staggering-cost-oftraining-sota-ai-models/ [April 2020].

[6] L.-X. Wang (1992), Fuzzy systems are universal approximators, IEEE International Conference on Fuzzy Systems, ISBN: 0-7803-0236-2, DOI: 10.1109/FUZZY.1992.258721.

[7] A. Dinu., L.P. Dinu, L.Franzoi, A.Sgarro (2018), Steinhaus Transforms of Fuzzy String Distances in Computational Linguistics, in: Medina J. et al. (eds) Information Processing and Management of Uncertainty in Knowledge-Based Systems. Theory and Foundations. IPMU 2018, Communications in Computer and Information Science, vol 853. Springer, Cham, ISBN 978-3-319-91472-5, DOI: https://doi.org/10.1007/978-3-319-91473-2_15.

[8] L. Franzoi (2018), Fuzzy information theory with applications to computational linguistics", $\mathrm{PhD}$ thesis, University of Bucharest.

[9] M. Aupetit, P. Couturier, P. Masote (2000), Function Approximation with Continuous SelfOrganizing Maps Using Neighboring Influence Interpolation, in: Proc. of Neural Computation (NC2000), Berlin, Germany, May 2000 http://citeseerx.ist.psu.edu/viewdoc/download?doi=10.1.1.16.320\&rep=rep1\&type=pdf.

[10] S. Ahmad, J. Hawkins (2015), Properties of Sparse Distributed Representations and their Application to Hierarchical Temporal Memory, arXiv:1503.07469v1.

[11] F. D. S. Webber, 2016, ,Semantic Folding Theory And its Application in Semantic Fingerprinting" arXiv:1511.08855v2. 